OPEN ACCESS

Edited by:

Stefano Caserta

University of Hull, United Kingdom

Reviewed by:

Behdad Afzali,

National Institute of Diabetes and Digestive and Kidney Diseases

(NIDDK), United States

Stephen Robert Daley,

Monash University, Australia

*Correspondence:

David Bending

d.a.bending@bham.ac.uk

Specialty section: This article was submitted to

Inflammation,

a section of the journal

Frontiers in Immunology

Received: 12 July 2018

Accepted: 12 September 2018

Published: 02 October 2018

Citation:

Copland A and Bending D (2018) Foxp3 Molecular Dynamics in Treg in

Juvenile Idiopathic Arthritis.

Front. Immunol. 9:2273.

doi: 10.3389/fimmu.2018.02273

\section{Foxp3 Molecular Dynamics in Treg in Juvenile Idiopathic Arthritis}

\author{
Alastair Copland and David Bending* \\ Institute of Immunology and Immunotherapy, College of Medical and Dental Sciences, University of Birmingham, \\ Birmingham, United Kingdom
}

Since the identification of the regulatory T-cell (Treg)-associated transcription factor Foxp3, there have been intensive research efforts to understand its biology and roles in maintaining immune homeostasis. It is well established that thymic selection of a repertoire of self-reactive Foxp3 ${ }^{+}$T-cells provides an essential mechanism to minimize reactions to self-antigens in the periphery, and thus aid in the prevention of autoimmunity. It is clear from both genetic and immunological analyses of juvenile idiopathic arthritis (JIA) patients that T-cells have a strong role to play in both the initiation and propagation of disease. The current paradigm is to view autoimmunity as a consequence of an imbalance between inflammatory and immunoregulatory mechanisms. This view has led to the assigning of cells and inflammatory mediators to different classes based on their assumed pro- or anti-inflammatory roles. This is typically reported as ratios of effector T-cells to Treg cells. Problematically, many analyses are based on static "snapshots-in-time," even though both mouse models and human patient studies have highlighted the dynamic nature of Foxp3 ${ }^{+}$T-cells in vivo, which can exhibit plasticity and time-dependent functional states. In this review, we discuss the role of Foxp3 dynamics in the control of T-cell responses in childhood arthritis, by reviewing evidence in humans and relevant mouse models of inflammatory disease. Whilst the cellular dynamics of Treg have been well evaluated-leading to standard data outputs such as frequency, quantity and quality (often assessed by in vitro suppressive capacity) - we discuss how recent insights into the molecular dynamics of Foxp3 transcription and its post-translational control may open up tantalizing new avenues for immunotherapies to treat autoimmune arthritis.

Keywords: Foxp3, juvenile idiopathic arthritis (JIA), transcriptional dynamics, Tocky, Treg

\section{AIMS AND SCOPE}

The aim of this article is to provide an overview of the literature reporting Foxp $3^{+}$Regulatory T cell (Treg) cell biology in juvenile idiopathic arthritis (JIA) and place this in the context of recent advances in understanding basic Treg biology. For review of JIA Treg biology, a defined Pubmed search was performed with the following terms: "Treg" OR "Foxp3" OR "Regulatory T cell" AND "Juvenile Idiopathic Arthritis"1. What is clear from these papers is that there has been great industry in elucidating Treg and effector T-cell biology and relating this to disease mechanisms from cellular viewpoints. This article aims to build on this body of knowledge by detailing how recent new

\footnotetext{
${ }^{1}$ Search date July $10^{\text {th }} 2018$.
} 
approaches are giving fresh insight into the molecular control of Foxp3 and the dynamics of T-cell regulation. It is hoped that this approach may help human immunologists to disambiguate markers used to identify Treg [e.g., CD25 and CD127 expression (1)] and stimulate fresh thinking about Foxp3mediated regulatory mechanisms in JIA.

\section{INTRODUCTION}

Foxp3 is essential for T-cell homeostasis and is considered one of the main drivers of Regulatory T-cell (Treg) differentiation (2). Mutations in the FOXP3 gene in humans cause the complex multiorgan autoimmune disease, Immune Dysregulation Polyendocrinopathy Enteropathy X-linked syndrome (IPEX) (3). This condition parallels the Scurfy mouse (4), where a two base-pair insertion in the murine Foxp3 gene results in a truncated form of Foxp3 protein (5) and loss of immune regulation. Scurfy mice typically die within 3 weeks, highlighting that loss of function of Foxp3 is not compatible with long-term survival. A Treg lineage (6) interpretation of these findings is that Foxp3 mutations lead to loss of a dedicated line of suppressor Tcells, presumably because Foxp 3 can no longer imprint the Treg suppressive phenotype (7). Treg are often divided into thymic and peripheral subsets, based on their sites of differentiation. Thymic Treg are self-reactive, and are important components of central tolerance. Peripheral Treg are thought to arise in response to innocuous antigens, and may be important for tolerance to dietary antigens or commensal bacteria. Together these subsets work to exert dominant tolerance to both self and foreign antigens (8).

Given the importance of Foxp3 to immune homeostasis and Treg biology, it has become a focal point of immunological research into diseases arising due to dysregulated T-cell responses, such as JIA. JIA is the most common form of autoimmune rheumatic disease with a prevalence in the region of one in a thousand in children under 16 years of age (9). JIA is a heterogeneous group of conditions, covering all forms of arthritis commencing in children under 16 years of age and lasting for at least 6 weeks duration (10). Although heterogeneity represents a challenge to translate basic immunological findings from animal models to JIA, access to the site of inflammation provides precious material for studying underlying immunological mechanisms.

\section{GENETICS OF JIA POINT TO T-CELL REGULATION}

Genetic association studies of JIA (incorporating the most common subtypes of disease) clearly highlight a key role for genes involved in the immune system, particularly those involved in the regulation of T-cell biology (11). Unsurprisingly, the strongest association is with human leukocyte antigen (HLA) alleles. This is a common occurrence in autoimmune diseases (12), which may be due to the finding that self-antigen-specific Treg selection is HLA allele-dependent and modulates susceptibility to autoimmunity (13). There also exists a striking association with genes involved in the molecular control of Treg biology. Although nine single nucleotide polymorphisms (SNPs) within the human FOXP3 gene show no significant associations to JIA (14) (suggesting these do not impact on FOXP3 in JIA), a key FOXP3-binding partner, Runt-related transcription factor 1 (RUNX1), is, however, significantly associated. In non-Foxp3 expressing T-cells, RUNX1 enhances interleukin (IL)2 expression through direct binding of the IL2 promoter in activated CD4 ${ }^{+}$ T-cells (7). However, in the context of Foxp3 expression, functional studies in mice have shown that Runx1 and Foxp3 form molecular complexes which lead to the repression of Il2 and interferon gamma (Ifng), and upregulation of Treg effector molecules, such as CD25 and cytotoxic lymphocyte antigen 4 (CTLA-4) (7). Thus, Foxp3 can re-direct the molecular machinery involved in T-cell activation in order to drive a Tcell-intrinsic suppressive programme. Whilst Foxp3 expression has been mainly studied in the context of Treg generation (15), the T-cell-intrinsic functions of FOXP3 expression during either T-cell activation (which is considered transient, but can have functional consequences for effector T-cells (16)) or peripheral (p)Treg generation in JIA remain unknown.

In addition to $R U N X 1$, genes encoding proteins involved in cytokine signaling pathways that are critical to the development of $\mathrm{FOXP}^{+}$T-cells are also significantly associated. IL2RA [gene encoding CD25, the original Treg marker (17)] and the IL2/IL21 cytokine locus display significant disease associations (11). Furthermore, deficiency in the IL-2 signaling molecule signal transducer and activator of transcription (STAT) $5 \mathrm{~b}$ has been reported in patients with JIA (18). IL-2 signaling is critical for the survival and fitness of Treg in the periphery (19) and is sensed by the conserved non-coding sequence 2 (CNS2) of the Foxp3 gene to maintain Treg cell identity (20). These findings suggest that molecular tuning of Foxp 3 transcription and function not only have genetic associations but may represent a new avenue through which to further our understanding of the pathogenesis of JIA.

\section{FOXP3+ ${ }^{+}$TREG CELL BIOLOGY IN JIA}

Analysis of Treg cells identified by CD25 expression has shown that such cells are enriched at the site of inflammation in JIA (21). The authors further dissected whether CD25 ${ }^{+}$T-cells show any relationship to disease severity. Here analysis of the oligoarticular (O)-JIA subset of patients is very useful since the O-JIA patient group can have divergent clinical outcomes (22). The "persistent" O-JIA subtype presents with a mild, remitting form of disease, which can spontaneously resolve. However, there exists a subset of O-JIA patients in which the disease progresses and arthritis extends to include an increasing number of joints, which are referred to as extended O-JIA patients. These divergent forms of O-JIA have allowed translational immunologists to compare between different severities of disease, which has proven powerful for correlating the role of Treg and T-helper subsets in JIA with measurable disease outcomes. Interestingly, CD25 ${ }^{+}$Treg cells are present in increased numbers within the joints of JIA patients with the milder persistent form of O-JIA compared to those 
with the more severe form of extended O-JIA disease (21). These findings were further verified using the "gold standard" Treg marker, FOXP3, in a separate patient cohort (23). Interestingly, both of these studies highlighted that compared to Treg in blood, Treg at the inflamed site were not only increased in proportion but they also displayed increased FOXP3 protein expression at the single cell level (24). These seemingly paradoxical findings of a vastly increased Treg signature at inflamed sites suggest that Treg presence alone may not detail the whole picture. Indeed, many subsequent studies have highlighted that the balance of effector and regulatory mechanisms may be a major influencer of disease outcome. Evidence to support this in JIA comes from findings that Th17 cells, which drive chronic arthritis via a cellular cascade (25), are also increased in the synovial fluid (SF) of JIA patients and display a reciprocal relationship with Treg (23) or FOXP3 expression levels (24).

Alterations in Treg phenotypes are likely influenced by the joint environment $(26,27)$. Firstly, Treg from SF are susceptible to the downregulation of FOXP3 following removal from the inflamed environment (26), which can be prevented by addition of synovial fluid to cultures. Furthermore, analysis of thymic Treg output [which has been shown to be altered in adult rheumatic disease (28)] revealed that JIA patients are no different from controls (29), suggesting that the quantity of thymic Treg generation may be unaffected. Furthermore functional studies on Treg from JIA patients suggest no qualitative difference in their ability to suppress T-cell responses (30).

As to what may explain disease in the presence of increased Treg frequency, investigators have looked at the effector T-cells. In vitro suppression assays have shown that Treg from the blood and SF of JIA patients display similar abilities in regulating the proliferation of conventional T-cells (Tconv) from the peripheral blood environment $(30,31)$. However, Tconv from the inflamed site showed resistance to suppression by Treg. Neat follow up in vitro studies to this have gone on to show that this resistance to suppression could be overcome by the blocking of inflammatory cytokines such as tumor necrosis factor alpha $(\mathrm{TNF} \alpha)(32,33)$, which may provide a potential mechanism of action for biologics used to treat JIA. However, whilst these studies suggest that SF Tconv may be more resistant to regulation in vitro, it is unknown whether Tconv are resistant to regulation in vivo. In particular, analysis of $\mathrm{Ki}^{+}{ }^{+}$(marker of cell proliferation) T-cells in the joints of JIA patients suggests a more complex picture, since the majority of dividing cells in the joint are Treg or Treglike [as evidenced by hypomethylation of the Foxp3 gene (27)]. Nonetheless a consensus has built based on the aforementioned work that site-specific Treg and Tconv interactions play central roles in the pathogenesis of JIA (Figure 1).

\section{DYNAMICS AND CLONAL RELATIONSHIPS BETWEEN FOXP3 ${ }^{+}$AND FOXP3- $^{-}$T-CELLS IN JIA}

Recent advances in sequencing technologies has allowed the profiling of the T-cell receptor (TCR) repertoires of Treg in JIA, which can be used to assess the relative clonal sharing

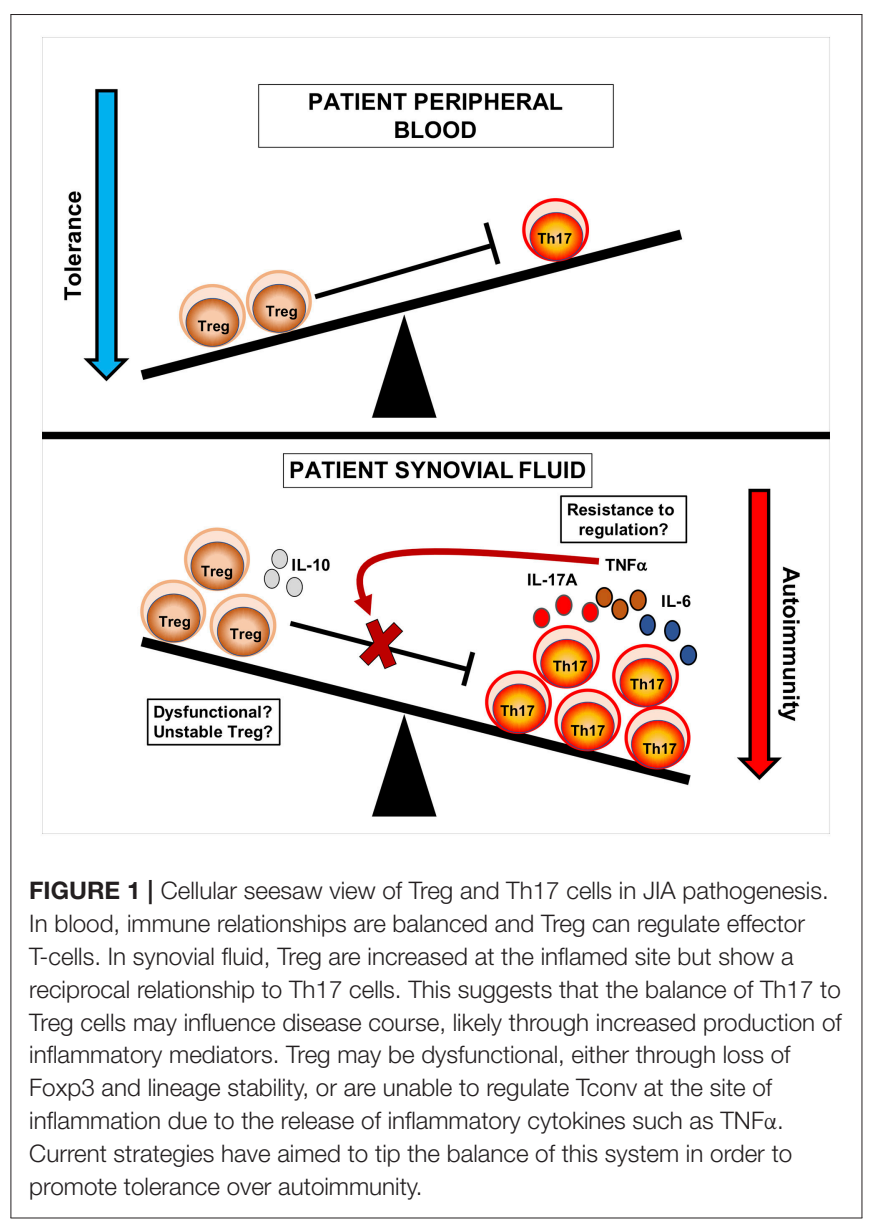

between different $\mathrm{CD} 4^{+} \mathrm{T}$-cell subsets and provide great insight into cellular dynamics. Deep sequencing of TCR $\beta$ chains has highlighted more restricted and oligoclonal repertoires in SF Tcells compared to blood $(26,34)$. Comparison between controls and JIA peripheral blood Treg suggests that JIA patients have skewing of both blood and SF TCR repertoires (34). This skewing of the repertoire could arise from recirculation of T-cells between blood and SF (35), but potentially could be an important biomarker for treatment. Indeed, animal studies have shown that bone marrow transplantation following peptidoglycaninduced arthritis revealed that Treg from the graft repopulate the immune system and show improved repertoire diversity (36). In fact, transfer of additional Foxp3-GFP ${ }^{+}$T-cells together with the bone marrow transfer graft did not induce additional clinical improvement but moreover delayed TCR repertoire diversification, cautioning the use of Treg transfers in such settings. This is likely due to the fact that these additional Treg suppress T-cell proliferation, thus delaying expansion of donor-derived T-cells. In addition, the authors were able to show that JIA patients undergoing haematopoietic stem cell transfers also showed improved Treg diversity. These findings suggest that an immune reset provides an opportunity for graftderived cells to regulate the autoimmune response, likely due to alterations in T-cell dynamics. Indeed, the SF environment 
appears to drive dynamic changes in Treg expression of key molecules. TCR repertoire analysis of SF T-cells expressing different combinations of CD25 and FOXP3 have shown that there is remarkable clonal sharing between $\mathrm{CD}_{2} 5^{+} \mathrm{FOXP}^{-}$, $\mathrm{CD}_{25}{ }^{+} \mathrm{FOXP}^{+}$, and $\mathrm{CD} 25^{-} \mathrm{FOXP}^{+}{ }^{+}$T-cells within the joint (26). These findings suggest that CD25 and FOXP3 expression within Treg-like populations may be dynamic within the joint; however, this does not preclude the possibility that CD25+FOXP3- T-cells also contain recently activated effector Tcells. Indeed, a thorough dissection of these populations would give useful insight to the dynamics of T-cell activation and FOXP3 expression in JIA. Given this observation, it will be intriguing for future studies to interrogate relationships between the TCR repertoires of Th17 and Treg cells within the joints of O-JIA patients, in order to establish whether the reciprocal relationship arises from discrete clones, or whether Th17/Treg plasticity is an important mechanism. For instance hybrid IL$17 \mathrm{~A}^{+} \mathrm{FOXP}^{+}$Treg, identified by expression of CD161, are significantly enriched at the site of inflammation $(37,38)$, and repertoire overlap between $\mathrm{CD}_{161}{ }^{+}$Treg and Tconv as a proportion of $\mathrm{CD}_{161}{ }^{+} \mathrm{SF}$ Treg was in the region of 20$30 \%$ (38), suggesting plasticity of T-cell responses within the joint. In summary, what these papers clearly show is that the joint environment is highly dynamic, and that snapshot in time analyses may obscure observations that may be accounted for by cellular and molecular dynamics.

\section{MOLECULAR DYNAMICS REGULATE FOXP3+ T-CELL BIOLOGY}

Whilst Foxp3 expression is used to define Treg subsets and is considered a stable marker of Treg cells, the clonal relationships between Treg and Tconv by TCR sequencing can also be explained by taking a molecular perspective (6). For instance, we have recently generated a new reporter system called Timer of cell kinetics and activity (Tocky) [(39); Figure 2A], which highlights a feedback control role for Foxp3 in regulating T-cell responses. This approach places the emphasis on the molecular dynamics of the system, with a reduced focus on cellular categorization, which can unnecessarily constrain analysis, particularly for highdimensional data sets.

\section{TIMER OF CELL KINETICS AND ACTIVITY (TOCKY) SYSTEM}

Tocky reporter system uses a short-lived fluorescent Timer protein (40) to capture the activity of the Foxp3 gene. Timer protein exhibits an initial blue fluorescent form, with an approximate half-life of $4 \mathrm{~h}$ in Foxp3-Tocky mice. Timer protein undergoes spontaneous and irreversible maturation into a red fluorescent form which has a half-life in the region of 5 days (41). Foxp3-Tocky therefore allows the detection of biologically important Foxp3 gene settings. New Foxp3 expressers are identified in Foxp3-Tocky mice by virtue of their pure blue fluorescence upon expression of the Timer gene, which is under the control of the Foxp3 gene regulatory elements. This system captures rapid changes in Foxp3 gene settings in response to immunological cues, giving insight to Foxp3 gene regulation at the level of hours vs. days. In contrast, fate-mapping approaches, which have largely suggested at a cellular level Treg cells are stable $(42,43)$, do not capture the "real-time" changes in Foxp3 gene activity which may greatly influence Treg function (41).

\section{FOXP3-TOCKY REVEALS EFFECTOR TREG DIFFERENTIATION AND IDENTIFIES PTREG}

We have revealed that mature Foxp3 expressers tune their Foxp3 gene setting to a temporally persistent state to control the resolution of skin inflammation. Thus, our recent data suggest that studying Foxp3 as a binary marker (i.e., to identify Treg and non-Treg) can lose biological information. Upon immunization we observed dramatic changes in the activity of Foxp3 transcription in various T-cell populations. At sites of inflammation (which are the relevant comparison for JIA) Treg increase transcription of Foxp3 in a Foxp3-protein dependent fashion (41). This form of Foxp3 autoregulation is key to driving what has been previously called the effector (e)Treg response, where cells display enhanced expression of immunoregulatory molecules, such as IL-10 and CTLA4. Thus, purely based on a molecular readout-the activity of the Foxp3 gene-we could identify the major features of previously coined "quiescent" Treg and effector (e)Treg subsets (Figure 2).

Foxp3-Tocky mice allow visualization of the earliest stages of $\mathrm{p}$ Treg development during physiological T-cell responses. We reported an increased proportion of T-cells acquire de novo Foxp3 expression within inflamed skin compared to noninflamed sites, during contact hypersensitivity. The biological significance of this is still to be fully determined, but we propose that induction of Foxp3 may be an important part of the resolution of $\mathrm{T}$-cell responses through the intrinsic regulation of Tconv $(16,41)$.

\section{POST-TRANSLATIONAL CONTROL OF FOXP3}

Aside from the transcriptional control of Foxp3, insight into the post-translational control of Foxp3 protein has been revealed over the past few years (44). Foxp3 protein has been shown to be polyubiquitinated at multiple lysine residues, which can lead to its proteasome-mediated degradation $(44,45)$. This process appears to be regulated by the activity of two enzymes, the deubiquitinase USP7 (44) and E3 ubiquitin ligase STUB1 (45). Here the authors of the studies were able to show that inflammatory cytokines, such as IL-6 [which is elevated in JIA synovial fluid (46)] could repress USP7 expression, resulting in increased turnover of Foxp3 and loss of Treg control of inflammation. These findings have led to the suggestion that small molecular inhibitors of this process could improve the stabilization of Foxp3 protein in cells, and therefore their functions (47). 


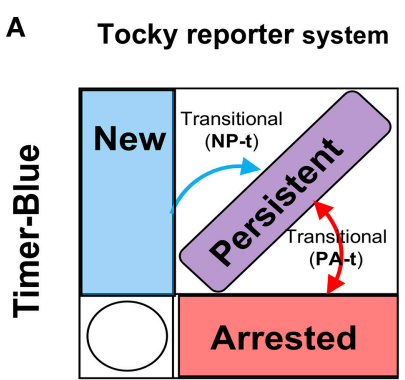

Timer-Red
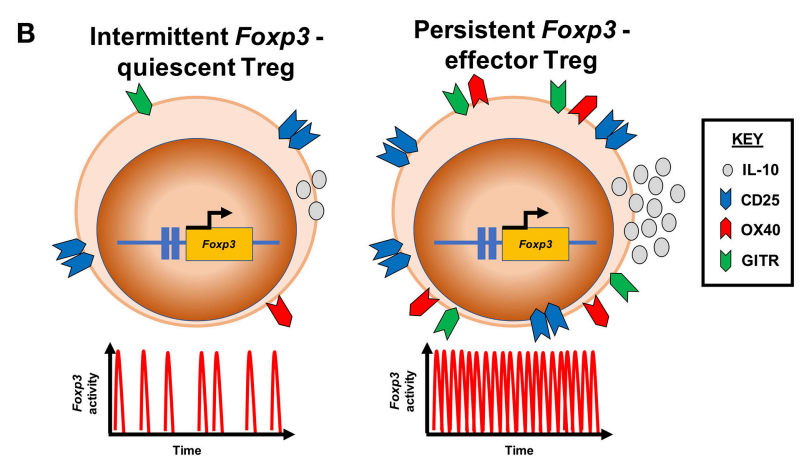

FIGURE 2 | Transcriptional dynamics of Foxp3 define functional Treg profiles. (A) The Tocky system [adapted from (39)] identifies different temporal expression patterns of the Foxp3 gene. Here a schematic is shown, illustrating how the Tocky system's timer locus approach can be used to identify different dynamics of Foxp3 expression based on the position of cells within a theoretical flow cytometric Timer-blue and Timer-red two-dimensional space: New expressers are pure blue, gradually acquiring red fluorescence after $4-8 \mathrm{~h}$, and moving to the New-Persistent transitional (NP-t) locus. Cells which continually transcribe Foxp3, accumulate in the blue ${ }^{+}$red $^{+}$diagonal, called Persistent. And cells that have recently ceased Foxp3 transcription are located in the pure red Arrested locus. As Foxp3 gene activity changes, cells can move between Arrested and Persistent zones within the Persistent-Arrested transitional (PA-t) locus. (B) Summary of Treg Foxp3 gene dynamics under different immunological contexts. In steady state, Treg exhibit intermittent Foxp3 gene activity, and express lower levels of CD25, GITR, OX40, and IL-10. Upon immune challenge, responsive Treg increase their Foxp3 transcriptional activity to a temporally persistent dynamic, which drives the Treg effector functions.

\section{FOXP3 MOLECULAR “TUNING” FOR THERAPY}

Foxp $3^{+}$T-cells have a large number of molecules that have been proposed as surrogate markers, such as high CD25 expression and low CD127 (1) (the IL-7 receptor alpha chain). In addition, they have also been shown to express high levels of the Tumor necrosis factor receptor superfamily members, which are important for their thymic development (48). Indeed, it has been known for over 15 years that glucocorticoid-induced TNFR-related protein (GITR) is a marker of thymic $\mathrm{CD}_{4}^{+}$ $\mathrm{CD}_{2} 5^{+}$subsets and targeting this membrane receptor with a monoclonal antibody could alter the course of autoimmunity (49). Using the Foxp3-Tocky tool, we have been able to give a dynamic perspective to common Treg cell surface markers (41). We were able to classify membrane receptors according to their relationship to Foxp 3 transcriptional activity, revealing that they can be classified into two main groups. Group I [containing amongst others TNFRII, C-C chemokine receptor type four (CCR4), CCR5] are high on activated T-cells and new Foxp3 expressers and remain high whilst active Foxp 3 transcription occurs. We could show that the targeting of a marker within this group (TNFRII) was able to increase the proportion of T-cells acquiring new Foxp 3 expression. Group II (containing amongst others CD25, OX40, and GITR) membrane receptors appeared to parallel the activity of Foxp 3 transcription and increased as Tcells moved into persistent dynamics of Foxp 3 Transcription (i.e., eTreg phenotype). Very interestingly, expression levels of these molecules fell considerably in T-cells with low or arrested Foxp3 transcriptional activity, implying they are selective to the eTregtype programme. OX40 was one member of this group, and we showed that upon anti-OX40 treatment Foxp $3^{+}$T-cells were shorter lived and "persistent" Foxp3 transcribers were reduced. This correlated with a delay in the resolution of allergic Tcell driven skin inflammation (41). Although these effects were modest, and the precise mechanisms of action remain to be fully elucidated, they show proof of concept that Foxp 3 transcriptional dynamics within T-cell populations can be modulated with measurable changes in disease outcomes.

\section{CONCLUSIONS AND FUTURE DIRECTIONS}

This review has highlighted the increases in our understanding of Foxp $3^{+}$T-cell biology in JIA. Recent work revealing how molecular pathways regulate Foxp3 protein and Foxp3 transcription should spur researchers to consider how these findings may be translated to human disease settings. For instance, it will be useful to determine whether the markers identified in basic animal models also hold equally true for humans. This could provide biomarkers to better understand the immunological effects of biologics for the treatment of autoimmune disorders such as JIA. In addition, given that Foxp3 protein can autoregulate its transcription, it will be very interesting to see whether post-translational modifications of Foxp3 can alter the Foxp3-driven autoregulatory loop (41, 50), and therefore enhance the Foxp3-driven T-cell programme. To take these ideas forward, however, the field may need to take a few steps back from the cell lineage paradigm and consider how the molecular dynamics of Foxp3-driven biology may provide new avenues for translational research in JIA.

\section{AUTHOR CONTRIBUTIONS}

$\mathrm{DB}$ conceived the review. $\mathrm{AC}$ and $\mathrm{DB}$ reviewed the literature and co-wrote the manuscript.

\section{FUNDING}

Work funded by a University of Birmingham Fellowship. 


\section{REFERENCES}

1. Liu W, Putnam AL, Xu-Yu Z, Szot GL, Lee MR, Zhu S, et al. CD127 expression inversely correlates with FoxP3 and suppressive function of human CD4+ T reg cells. J Exp Med. (2006) 203:1701-11. doi: 10.1084/jem.20060772

2. Sakaguchi S, Yamaguchi T, Nomura T, Ono M. Regulatory T cells and immune tolerance. Cell (2008) 133:775-87. doi: 10.1016/j.cell.2008.05.009

3. Bennett CL, Christie J, Ramsdell F, Brunkow ME, Ferguson PJ, Whitesell L, et al. The immune dysregulation, polyendocrinopathy, enteropathy, X-linked syndrome (IPEX) is caused by mutations of FOXP3. Nat Genet. (2001) 27:20-1. doi: 10.1038/83713

4. Lyon MF, Peters J, Glenister PH, Ball S, Wright E. The scurfy mouse mutant has previously unrecognized hematological abnormalities and resembles Wiskott-Aldrich syndrome. Proc Natl Acad Sci USA. (1990) 87:2433-7.

5. Brunkow ME, Jeffery EW, Hjerrild KA, Paeper B, Clark LB, Yasayko SA, et al. Disruption of a new forkhead/winged-helix protein, scurfin, results in the fatal lymphoproliferative disorder of the scurfy mouse. Nat Genet. (2001) 27:68-73. doi: $10.1038 / 83784$

6. Ono M, Tanaka RJ. Controversies concerning thymus-derived regulatory T cells: fundamental issues and a new perspective. Immunol Cell Biol. (2016) 94:3-10. doi: 10.1038/icb.2015.65

7. Ono M, Yaguchi H, Ohkura N, Kitabayashi I, Nagamura Y, Nomura T, et al. Foxp3 controls regulatory T-cell function by interacting with AML1/Runx1. Nature (2007) 446:685-9. doi: 10.1038/nature05673

8. Lee HM, Bautista JL, Hsieh CS. Thymic and peripheral differentiation of regulatory $\mathrm{T}$ cells. Adv Immunol. (2011) 112:25-71. doi: 10.1016/B978-0-12-387827-4.00002-4

9. Manners PJ, Bower C. Worldwide prevalence of juvenile arthritis why does it vary so much? J Rheumatol. (2002) 29:1520-30.

10. Ravelli A, Martini A. Juvenile idiopathic arthritis. Lancet (2007) 369:767-78. doi: 10.1016/S0140-6736(07)60363-8

11. Hinks A, Cobb J, Marion MC, Prahalad S, Sudman M, Bowes J, et al. Dense genotyping of immune-related disease regions identifies 14 new susceptibility loci for juvenile idiopathic arthritis. Nat Genet. (2013) 45:664-9. doi: $10.1038 / n g .2614$

12. Gough SC, Simmonds MJ. The HLA region and autoimmune disease: associations and mechanisms of action. Curr Genomics (2007) 8:453-65. doi: 10.2174/138920207783591690

13. Ooi JD, Petersen J, Tan YH, Huynh M, Willett ZJ, Ramarathinam SH, et al. Dominant protection from HLA-linked autoimmunity by antigen-specific regulatory T cells. Nature (2017) 545:243-7. doi: 10.1038/nature22329

14. Eastell T, Group BS, Hinks A, Thomson W. SNPs in the FOXP3 gene region show no association with juvenile idiopathic arthritis in a UK Caucasian population. Rheumatology (2007) 46:1263-5. doi: 10.1093/rheumatology/kem129

15. Gavin MA, Rasmussen JP, Fontenot JD, Vasta V, Manganiello VC, Beavo JA, et al. Foxp3-dependent programme of regulatory T-cell differentiation. Nature (2007) 445:771-5. doi: 10.1038/nature05543

16. McMurchy AN, Gillies J, Gizzi MC, Riba M, Garcia-Manteiga JM, Cittaro D, et al. A novel function for FOXP3 in humans: intrinsic regulation of conventional $\mathrm{T}$ cells. Blood (2013) 121:1265-75. doi: 10.1182/blood-2012-05-431023

17. Sakaguchi S, Sakaguchi N, Asano M, Itoh M, Toda M. Immunologic selftolerance maintained by activated T cells expressing IL-2 receptor alphachains (CD25). Breakdown of a single mechanism of self-tolerance causes various autoimmune diseases. J Immunol. (1995) 155:1151-64.

18. Kanai T, Jenks J, Nadeau KC The STAT5b pathway defect and autoimmunity. Front Immunol. (2012) 3:234. doi: 10.3389/fimmu.2012.00234

19. Fontenot JD, Rasmussen JP, Gavin MA, Rudensky AY. A function for interleukin 2 in Foxp3-expressing regulatory T cells. Nat Immunol. (2005) 6:1142-51. doi: $10.1038 /$ ni1263

20. Feng Y, Arvey A, Chinen T, van der Veeken J, Gasteiger G, Rudensky AY, Control of the inheritance of regulatory $\mathrm{T}$ cell identity by a cis element in the Foxp3 locus. Cell (2014) 158:749-63. doi: 10.1016/j.cell.2014.07.031

21. de Kleer IM, Wedderburn LR, Taams LS, Patel A, Varsani H, Klein M, et al. CD4+CD25bright regulatory $\mathrm{T}$ cells actively regulate inflammation in the joints of patients with the remitting form of juvenile idiopathic arthritis. J Immunol. (2004) 172:6435-43. doi: 10.4049/jimmunol.172.10.6435
22. Macaubas C, Nguyen K, Milojevic D, Park JL, Mellins ED. Oligoarticular and polyarticular JIA: epidemiology and pathogenesis. Nat Rev Rheumatol. (2009) 5:616-26. doi: 10.1038/nrrheum.2009.209

23. Nistala K, Moncrieffe H, Newton KR, Varsani H, Hunter P, Wedderburn LR. Interleukin-17-producing $\mathrm{T}$ cells are enriched in the joints of children with arthritis, but have a reciprocal relationship to regulatory T cell numbers. Arthritis Rheum. (2008) 58:875-87. doi: 10.1002/art. 23291

24. Olivito B, Simonini G, Ciullini S, Moriondo M, Betti L, Gambineri E, et al. Th17 transcription factor RORC2 is inversely correlated with FOXP3 expression in the joints of children with juvenile idiopathic arthritis. J Rheumatol. (2009) 36:2017-24. doi: 10.3899/jrheum.090066

25. Hirota $\mathrm{K}$, Hashimoto $\mathrm{M}$, Ito $\mathrm{Y}$, Matsuura $\mathrm{M}$, Ito $\mathrm{H}$, Tanaka $\mathrm{M}$, et al. Autoimmune Th17 cells induced synovial stromal and innate lymphoid cell secretion of the cytokine GM-CSF to initiate and augment autoimmune arthritis. Immunity (2018) 48:1220-32.e5. doi: 10.1016/j.immuni.2018.04.009

26. Bending D, Giannakopoulou E, Lom H, Wedderburn LR. Synovial regulatory T cells occupy a discrete TCR Niche in human arthritis and require local signals to stabilize FOXP3 protein expression. J Immunol. (2015) 195:5616-24. doi: 10.4049/jimmunol.1500391

27. Bending D, Pesenacker AM, Ursu S, Wu Q, Lom H, Thirugnanabalan $\mathrm{B}$, et al. Hypomethylation at the regulatory $\mathrm{T}$ cell-specific demethylated region in CD25hi $\mathrm{T}$ cells is decoupled from FOXP3 expression at the inflamed site in childhood arthritis. J Immunol. (2014) 193:2699-708. doi: 10.4049/jimmunol.1400599

28. Koetz K, Bryl E, Spickschen K, O’Fallon WM, Goronzy JJ, Weyand CM. T cell homeostasis in patients with rheumatoid arthritis. Proc Natl Acad Sci USA. (2000) 97:9203-8. doi: 10.1073/pnas.97.16.9203

29. Lorenzi AR, Morgan TA, Anderson A, Catterall J, Patterson AM, Foster HE, et al. Thymic function in juvenile idiopathic arthritis. Ann Rheum Dis. (2009) 68:983-90. doi: 10.1136/ard.2008.088112

30. Wehrens EJ, Mijnheer G, Duurland CL, Klein M, Meerding J, van Loosdregt $\mathrm{J}$, et al. Functional human regulatory $\mathrm{T}$ cells fail to control autoimmune inflammation due to $\mathrm{PKB} / \mathrm{c}$-akt hyperactivation in effector cells. Blood (2011) 118:3538-48. doi: 10.1182/blood-2010-12-3 28187

31. Haufe S, Haug M, Schepp C, Kuemmerle-Deschner J, Hansmann S, Rieber N, et al. Impaired suppression of synovial fluid CD4+CD25- T cells from patients with juvenile idiopathic arthritis by CD4+CD25+ Treg cells. Arthritis Rheum. (2011) 63:3153-62. doi: 10.1002/art.30503

32. Petrelli A, Wehrens EJ, Scholman RC, Prakken BJ, Vastert SJ, van Wijk F, Self-sustained resistance to suppression of CD8+ Teff cells at the site of autoimmune inflammation can be reversed by tumor necrosis factor and interferon-gamma blockade. Arthritis Rheumatol. (2016) 68:229-36. doi: $10.1002 /$ art.39418

33. Wehrens EJ, Vastert SJ, Mijnheer G, Meerding J, Klein M, Wulffraat $\mathrm{NM}$, et al. Anti-tumor necrosis factor alpha targets protein kinase $\mathrm{B} / \mathrm{c}$-Akt-induced resistance of effector cells to suppression in juvenile idiopathic arthritis. Arthritis Rheum. (2013) 65:3279-84. doi: 10.1002/art. 38132

34. Henderson LA, Volpi S, Frugoni F, Janssen E, Kim S, Sundel RP, et al. Nextgeneration sequencing reveals restriction and clonotypic expansion of treg cells in juvenile idiopathic arthritis. Arthritis Rheumatol. (2016) 68:1758-68. doi: 10.1002/art.39606

35. Rossetti M, Spreafico R, Consolaro A, Leong JY, Chua C, Massa M, et al. TCR repertoire sequencing identifies synovial Treg cell clonotypes in the bloodstream during active inflammation in human arthritis. Ann Rheum Dis. (2017) 76:435-41. doi: 10.1136/annrheumdis-2015-208992

36. Delemarre EM, van den Broek T, Mijnheer G, Meerding J, Wehrens EJ, Olek $\mathrm{S}$, et al. Autologous stem cell transplantation aids autoimmune patients by functional renewal and TCR diversification of regulatory T cells. Blood (2016) 127:91-101. doi: 10.1182/blood-2015-06-649145

37. Pesenacker AM, Bending D, Ursu S, Wu Q, Nistala K, Wedderburn LR. CD161 defines the subset of FoxP3+ $\mathrm{T}$ cells capable of producing proinflammatory cytokines. Blood (2013) 121:2647-58. doi: 10.1182/blood-2012-08-443473

38. Duurland CL, Brown CC, O'Shaughnessy RF, Wedderburn LR. CD161(+) Tconv and CD161(+) Treg share a transcriptional and functional phenotype 
despite limited overlap in TCRbeta repertoire. Front Immunol. (2017) 8:103. doi: 10.3389/fimmu.2017.00103

39. Bending D, Martin PP, Paduraru A, Ducker C, Marzaganov E, Laviron $\mathrm{M}$, et al. A timer for analyzing temporally dynamic changes in transcription during differentiation in vivo. J Cell Biol. (2018) 217:2931-50. doi: $10.1083 /$ jcb. 201711048

40. Subach FV, Subach OM, Gundorov IS, Morozova KS, Piatkevich KD, Cuervo AM, et al. Monomeric fluorescent timers that change color from blue to red report on cellular trafficking. Nat Chem Biol. (2009) 5:118-26. doi: 10.1038/nchembio.138

41. Bending D, Paduraru A, Ducker CB, Prieto Martin P, Crompton T, Ono M, A temporally dynamic Foxp3 autoregulatory transcriptional circuit controls the effector Treg programme. EMBO J. (2018) 37:e99013. doi: 10.15252/embj.201899013

42. Rubtsov YP, Niec RE, Josefowicz S, Li L, Darce J, Mathis D, et al. Stability of the regulatory T cell lineage in vivo. Science (2010) 329:1667-71. doi: $10.1126 /$ science. 1191996

43. Miyao T, Floess S, Setoguchi R, Luche H, Fehling HJ, Waldmann H, et al. Plasticity of Foxp3(+) T cells reflects promiscuous Foxp3 expression in conventional $\mathrm{T}$ cells but not reprogramming of regulatory $\mathrm{T}$ cells. Immunity (2012) 36:262-75. doi: 10.1016/j.immuni.2011.12.012

44. van Loosdregt J, Fleskens V, Fu J, Brenkman AB, Bekker CP, Pals CE, et al. Stabilization of the transcription factor Foxp3 by the deubiquitinase USP7 increases Treg-cell-suppressive capacity. Immunity (2013) 39:259-71. doi: 10.1016/j.immuni.2013.05.018

45. Chen Z, Barbi J, Bu S, Yang HY, Li Z, Gao Y, et al. The ubiquitin ligase Stub1 negatively modulates regulatory $\mathrm{T}$ cell suppressive activity by promoting degradation of the transcription factor Foxp3. Immunity (2013) 39:272-85. doi: 10.1016/j.immuni.2013.08.006
46. de Jager W, Hoppenreijs EP, Wulffraat NM, Wedderburn LR, Kuis W, Prakken BJ. Blood and synovial fluid cytokine signatures in patients with juvenile idiopathic arthritis: a cross-sectional study. Ann Rheum Dis. (2007) 66:589-98. doi: 10.1136/ard.2006.061853

47. van Loosdregt J, Coffer PJ. Post-translational modification networks regulating FOXP3 function. Trends Immunol. (2014) 35:368-78. doi: $10.1016 /$ j.it.2014.06.005

48. Mahmud SA, Manlove LS, Schmitz HM, Xing Y, Wang Y, Owen DL, et al. Costimulation via the tumor-necrosis factor receptor superfamily couples TCR signal strength to the thymic differentiation of regulatory T cells. Nat Immunol. (2014) 15:473-81. doi: 10.1038/ni.2849

49. Shimizu J, Yamazaki S, Takahashi T, Ishida Y, Sakaguchi S. Stimulation of $\mathrm{CD} 25(+) \mathrm{CD} 4(+)$ regulatory $\mathrm{T}$ cells through GITR breaks immunological self-tolerance. Nat Immunol. (2002) 3:135-42. doi: 10.1038/ ni759

50. Zheng Y, Josefowicz S, Chaudhry A, Peng XP, Forbush K, Rudensky AY. Role of conserved non-coding DNA elements in the Foxp3 gene in regulatory T-cell fate. Nature (2010) 463:808-12. doi: 10.1038/nature08750

Conflict of Interest Statement: The authors declare that the research was conducted in the absence of any commercial or financial relationships that could be construed as a potential conflict of interest.

Copyright $\odot 2018$ Copland and Bending. This is an open-access article distributed under the terms of the Creative Commons Attribution License (CC BY). The use, distribution or reproduction in other forums is permitted, provided the original author(s) and the copyright owner(s) are credited and that the original publication in this journal is cited, in accordance with accepted academic practice. No use, distribution or reproduction is permitted which does not comply with these terms. 